\title{
Preparedness of Anesthesiologists Working in Humanitarian Disasters
}

\author{
Bernhard Rössler, MD, MIH, Peter Marhofer, MD, Michael Hüpfl, MD, \\ Bernadette Peterhans, RN, MPH, and Karl Schebesta, MD
}

\section{ABSTRACT}

Objective: Many skills needed to provide patients with safe, timely, and adequate anesthesia care during humanitarian crisis and disaster relief operations are not part of the daily routine before deployment. An exploratory study was conducted to identify preparedness, knowledge, and skills needed for deployment to complex emergencies.

Methods: Anesthesiologists who had been deployed during humanitarian crisis and disaster relief operations completed an online questionnaire assessing their preparedness, skills, and knowledge needed during deployment. Qualitative data were sorted by frequencies and similarities and clustered accordingly.

Results: Of 121 invitations sent out, 55 (46\%) were completed and returned. Of these respondents, 24\% did not feel sufficiently prepared for the deployment, and $69 \%$ did not undertake additional education for their missions. Insufficient preparedness involved equipment, drugs, regional anesthesia, and related management.

Conclusions: As the lack of preparation and relevant training can create precarious situations, anesthesiologists and deploying agencies should improve preparedness for anesthesia personnel. (Disaster Med Public Health Preparedness. 2013;7;408-412)

Key Words: anesthesia, emergency service, disasters, international agencies, relief work

$\mathrm{I}$ a disaster, the necessary response to serve the affected population overwhelms existing local health care capacities. ${ }^{1}$ In a Lancet publication by Salama and colleagues, the term complex emergency was defined as a situation in which mortality among the civilian population substantially increases above the population baseline. ${ }^{2}$ This situation occurs either as a result of the direct effects of the disaster or indirectly through the increased prevalence of malnutrition or the transmission of communicable diseases, or both. ${ }^{2}$

During the past few decades, an increasing number of national and international organizations have been deploying surgical and anesthesia teams to deliver necessary treatment for the affected population. ${ }^{3-7}$ Providing safe, timely, and adequate anesthesia care during such emergency and disaster relief operations is one of the most challenging situations for anesthesiologists.

Anesthesiologists working in disaster settings are required to perform high-quality anesthesia in either a field hospital, local clinic, or even outside a health facility. Furthermore, for many of the deployed anesthesiologists the setting, equipment, tasks, and morbidity patterns can be very different than in the normal place of work. In addition to the need for excellent medical knowledge, anesthesiologists have to be prepared for unfamiliar tasks such as readiness preparation; triage of mass casualties; a high numbers of crush, burn, or gunshot victims; limited access to resources; lack of postanesthesia and intensive care facilities; unfamiliar anesthesia techniques; and unfamiliar equipment that may need maintenance and repair. ${ }^{3,4,6,8-10}$

Anesthesiologists who normally practice in hightechnology surroundings will therefore require special training and preparation to respond adequately in disasters associated with natural hazards or settings of armed conflict. ${ }^{8}$ As a result, it is challenging for organizations recruiting medical staff for relief operations to find sufficient numbers of anesthesiologists with competence in managing the essential challenges during the mission.

Consequently, we hypothesized that preparedness is insufficient among anesthesiologists working in complex emergencies, disasters associated with natural hazards, or armed conflict. Therefore, perceived preparedness was defined as the primary outcome 
parameter. In addition, this trial evaluated participation in preparatory courses and aimed at identifying the skills and knowledge of anesthesiologists needed for deployment to these settings.

\section{METHODS}

A questionnaire based on the current literature and in cooperation with deploying organizations was designed to cover the following aspects of the anesthesiologists' work: type of mission, setting and workplace, type of surgeries and caseload, equipment, management, supply, and education and staff training. Questions were designed to evaluate demographics of the respondents and their perception on relevance of knowledge and skills in each subcategory during a relief operation. The participants were asked to specify skills and techniques required during deployment. Open, qualitative questions were included in the questionnaire to allow the participants to state personal experiences and necessary skills and knowledge specific to a mission.

This trial aimed at including some of the main deploying agencies from Europe. Consequently, the invitation to the web-based questionnaire was distributed via e-mail to anesthesiologists who have been deployed by the following organizations: Médecins Sans Frontières (MSF) Austria, MSF Switzerland, International Committee of the Red Cross (ICRC), Austrian Red Cross, German Red Cross, Finnish Red Cross, Go International, and Johanniter International. The questionnaire (www.surveymonkey.com/anaesthesiaincomplex emergencies) was available from May 1, 2011, until June 30, 2011.

To ensure confidentiality, the questionnaire was conducted anonymously and independently. For protection of privacy, e-mails were sent from the human resource representative of the participating organization. To increase participation, a reminder e-mail was sent 3 weeks after the initial invitation. Care was taken to minimize the risk of participants receiving the same invitation by various organizations. If more than 1 invitation was received, the anesthesiologists were instructed to answer only once and to focus on the last mission, where applicable. The authors expected a return rate of $30 \%$ by using an online questionnaire with a reminder e-mail. ${ }^{11}$ Qualitative data were sorted by frequencies and similarities and clustered according to type of answers.

Data management was conducted with Microsoft Excel for Mac 2011 (14.0.2). Statistical analysis was performed with SPSS 18.0 (IBM) for all analyses. Data were prepared as percent of respondents, quartiles, and ranges, as well as mean plus or minus the standard deviation, where appropriate.

\section{RESULTS}

In total, 121 invitations were sent. While $62(51 \%)$ answered the questionnaire, 7 were excluded from the final analysis due to incomplete answers. Consequently, 55 questionnaires
TABLE

$\begin{aligned} & \text { Demographic Data of the Respondents } \\ & \text { Category } \\ & \text { No. of Respondents }\end{aligned}$
$\begin{array}{lrr}\text { Age, y } & \% \\ 25-30 & 1 & \\ 31-40 & 11 & 1.8 \\ 41-50 & 15 & 20.0 \\ 51-60 & 14 & 27.3 \\ >60 & 14 & 25.5 \\ \text { Gender } & & 25.5 \\ \text { Female } & 29 & \\ \text { Male } & 26 & 52.7 \\ \text { Board certified duration, y } & & 47.3 \\ \text { 1 } & 3 & \\ 2-5 & 8 & 5.5 \\ \text { 6-10 } & 6 & 14.5 \\ >10 & 38 & 69.1 \\ \end{array}$

TABLE 2

\begin{tabular}{|c|c|c|}
\hline Category & No. of Respondents & $\%$ \\
\hline \multicolumn{3}{|c|}{ Previous deployments } \\
\hline 1 & 20 & 36.4 \\
\hline $2-5$ & 12 & 21.8 \\
\hline$>5$ & 23 & 41.8 \\
\hline \multicolumn{3}{|c|}{ Type of deployments (multiple answers) } \\
\hline Armed conflict & 37 & 67.3 \\
\hline Refugee camp & 8 & 14.5 \\
\hline Nuclear accident & 0 & 0.0 \\
\hline Natural disaster & 32 & 58.2 \\
\hline Other & 5 & 9.1 \\
\hline
\end{tabular}

entered data analysis. Demographic data of the respondents have been provided in Table 1 .

Twenty-six $(47.3 \%)$ respondents had been on a mission for the ICRC, 27 (49.1\%) for MSF, and 22 (40.0\%) for a National Red Cross or Red Crescent Society. Ten (18.2\%) respondents had been working for other organizations. Twenty-two anesthesiologists were working in Haiti (40.0\%) on their last deployment; 7 were in Pakistan (12.7\%); 5 in Afghanistan (9.1\%); 3 in Libya (5.5\%); 2 each in Sudan, Thailand, Chad, Democratic Republic of Congo, and Palestine (3.6\% each); and 1 each in Burundi, Indonesia, Ivory Coast, Nigeria, Ruanda, Russia, and Yemen (1.8\% each). One participant chose not to specify the country of deployment. Deployment details have been provided in Table 2 .

Thirteen respondents $(23.6 \%)$ did not feel sufficiently prepared for the mission, and $35(63.6 \%)$ respondents did not participate in a preparatory course covering anesthesia topics by the deploying organization before departure. The median duration of the course for the deploying organization 


\section{TABLE 3}

\section{Procedures Requiring Anesthesia}

Category $\%$

Trauma/orthopedic

Gunshot

Crush

Burn

Amputations

Obstetrics/gynecology

General surgery

Pediatrics

Neurosurgery

Cardiothoracic

Ear, nose, throat

Ophthalmologic

Other

was 4 days (range: 1 h-30 days). Furthermore, 38 (69.1\%) respondents did not complete additional training relevant for missions. Relevant additional training included public health, tropical medicine, humanitarian assistance, disaster medicine, health emergencies in large populations (HELP), advanced trauma live support (ATLS), and management courses.

Six $(30 \%)$ of the 20 respondents who took part in a preparatory course did not feel sufficiently prepared. Four participants perceived themselves as insufficiently prepared in the group after only 1 deployment before answering the questionnaire $(33.3 \%)$, and 4 participants with 2 to 5 previous deployments had the lowest rate of insufficient preparedness $(17.4 \%)$. In the group with more than 5 previous deployments, $5(25 \%)$ felt insufficiently prepared.

During their missions, $45(81.8 \%)$ anesthesiologists had to do maintenance work on anesthesia equipment, while 28 (50.9\%) had to repair anesthesia equipment. Surgical suite management had to be done by 32 (58.2\%) of the respondents; 28 (50.9\%) had to conduct triage of mass casualties; 25 (45.5\%) were responsible for shift schedules of nurses and physicians and for staff management. Ordering supplies, consumables, and machines was done by 31 (56.4\%) of the respondents.

Regarding the lack of specific information before departure, respondents emphasized the need for information on anesthesia topics relevant to a given setting or mission. Asked what important part of the deployment was not covered in the training in their home country, 9 respondents emphasized lack of proficiency to deal with equipment problems (eg, maintenance, repair of anesthetic machines, monitoring, or sterilizers). Six emphasized the lack of management skills, including administrative work such as maintaining a supply chain of anesthetic goods or supervision of local staff; while 5 respondents focused on unfamiliar medications or blood products (eg, halothane, ketamine, local anesthetics, or full blood packages). Another 5 respondents described the lack of regional anesthetic techniques, such as neuroaxial and peripheral techniques, landmark approach, and pediatric regional anesthesia. Three respondents were lacking teaching skills and materials to train local staff, while 2 emphasized a need for improved psychological preparation. Most commonly performed procedures requiring anesthetics have been listed in Table 3 .

\section{DISCUSSION}

Even though all of the respondents were experienced clinicians and more than two-thirds were board certified longer than 10 years, more than one-fifth did not feel sufficiently prepared for the deployment. In addition, the majority did not complete additional education relevant for missions. Respondents especially felt insufficiently prepared after their first deployment.

Job profiles from agencies deploying anesthesiologists often contain a broad field of expertise necessary for the applicant. While these descriptions are available online for many organizations, their existence alone does not inevitably create awareness and positive impact on preparedness. Improved preparation and transparency with regard to the expected duties are necessary. Nevertheless, lack of knowledge and skills was most frequently related to anesthesia and management topics as well as psychological preparation. The respondents perceived insufficient preparation, for example, regarding equipment and drugs.

This lack of knowledge can lead to precarious situations, because familiarity with anesthesia equipment is essential in the performance of safe, high-quality anesthesia and, consequently, the issue of adequate training and introduction, ${ }^{12,13}$ especially as medication errors are a major cause of anesthetic-related death. ${ }^{14,15}$ International standards for providing safe anesthesia, including the necessity of monitoring, have been published. Nonadherence to the latter, even in the most austere setting, must be regarded as highly questionable practice. ${ }^{16-18}$ Maintenance and repair was a crucial point often emphasized by the respondents. Every 4 in 5 respondents had to do maintenance work on anesthesia equipment, and more than one-half had to repair equipment. Because the lack of repair can lead to reduced surgical suite capacity and insufficient maintenance can dangerously decrease patients' safety, these shortcomings must be overcome. ${ }^{12,13}$

Furthermore, participants emphasized the lack of regional anesthesia skills. This finding was unexpected, as previous findings stressed the importance and value of adequate integration of regional anesthesia techniques in the care of trauma victims. ${ }^{4}$ Also, we found that some deploying organizations encourage the use of regional anesthesia techniques. ${ }^{19}$ Participants emphasized the need for adequate training. The reasons for the low utilization of peripheral blocks were unfamiliarity with the block or the application technique and difficulty in communication with the patient due to language barriers. However, the findings from Missair 
and colleagues were aligned with previous findings from Rice and co-workers, who described that regional anesthesia was extremely useful to provide operative anesthesia and postoperative analgesia while reducing the burden on the postanesthesia care unit. ${ }^{4,6}$ Regional anesthesia remains a safe but underused technique. Even though regional anesthesia is frequently performed using ultrasound-guided techniques in European teaching hospitals, ${ }^{20}$ anesthesiologists are not routinely trained in the landmark technique, as performing a block using the landmark technique exclusively, when ultrasound or nerve stimulator is available, is hardly justifiably.

Although some deployments use techniques and equipment well outside the standard care and training of the contracted anesthesiologists, training to use landmark techniques would be neither feasible (timing of training before deploying, skill retention between deployments) nor hardly justifiable in view of current developments. ${ }^{20}$ To benefit from the wide application of regional anesthesia techniques, especially in settings with a high number of traumatic injuries, deploying agencies should be strongly encouraged to provide the technical background to allow application. ${ }^{4}$

International anesthesia assistance has been designed to provide quality health care through uninterrupted support to local health care facilities, and therefore reducing the burden of disease of a population. ${ }^{21}$ Consequently, not only disasterrelated injuries are to be treated, but capacities have to also include a broad surgical spectrum of care for the population including, for example, general surgery and obstetric or pediatric anesthesia. Often nonsurgical conditions prove challenging for medical staff, and outbreaks of diarrheal diseases or measles also heavily affect the work of the anaesthesiologist. $^{22}$ The lack of knowledge in tropical diseases was noted. In addition, knowledge of infectious diseases and tropical medicine can be important, as during many missions anesthesiologists serve as the intensivist. Furthermore, knowledge of tropical medicine is essential, as populations that are normally affected by these diseases suddenly suffer from an outbreak threatening a large part of the population. ${ }^{23}$

Initial care of the patients on arrival was also the anesthesiologists' task in some cases. Triage of mass casualties was performed by more than one-half of the respondents. The majority of the respondents had to perform surgical suite and intensive care unit management, as indicated in job descriptions of deploying agencies. Intensive care treatment in emergency settings included sepsis, tetanus, burns, and obstetric and pediatric critical care, whether or not resources are limited.

Deployments after natural disasters often do not allow participation in extended preparatory courses once the organizations start to recruit staff. Consequently, anesthesiologists interested in international humanitarian work should be strongly encouraged to participate in relevant courses covering existing gaps in advance. Some longer term humanitarian disasters (eg, armed conflicts and refugee care) might also allow the deploying organization to conduct shortterm preparatory courses that focus on the anesthesiologists' work or to participate in a course run by a third party. Nevertheless, the heterogeneity of organizations, deployments, and settings would limit the impact of a single preparatory course. This situation is also reflected in the fact that respondents who completed relevant additional training often completed more than 1 course.

In situations of armed conflict, humanitarian assistance can be provided by military or civilian organizations. Great differences exist between civilian nongovernmental organizations and military medical services. However, our questionnaire aimed to elicit the civilian anesthesiologists' role in complex emergencies. Furthermore, services that have to be provided are directly related to preexisting, very heterogeneous, local health infrastructure and their capacities during crisis. Consequently, the questionnaire did not aim at identifying situations in which the lack of preparedness led to poor outcomes. The questionnaire focused on international medical staff of organizations and did not include local health workers and anesthesiologists from recipient countries.

While some respondents participated in preparatory courses covering anesthesia topics by the deploying organization, others, although sometimes deployed by the same organizations, did not. Whether the courses were not held before some deployments or the individual respondent could not attend was beyond the scope of this questionnaire. To reduce nonresponder or selection bias, reminder e-mails were sent through the participating organization. While a return rate more than $30 \%$ for web-based questionnaires was acceptable in comparison with previous publications, the results have to be interpreted carefully and the conclusions cannot be extrapolated to every deploying organization and even less so to all deployed anesthetists. Furthermore, we cannot rule out a remaining selection bias resulting from the choice of collaborating organizations and their current or previous employed anesthesiologists to participate. ${ }^{11}$

\section{CONCLUSIONS}

The findings from this study provide evidence that many anesthesiologists deployed to complex emergencies lack important preparedness before leaving. Gaps in contextspecific medical, technical, and administrational aspects must therefore be overcome, and additional research in this field is needed. Improved preparation in close collaboration with deploying agencies is necessary to provide safe anesthesia and must strongly be encouraged. Nevertheless, while striving for improved preparation, the format and optimal duration of a preparatory course aimed at filling this gap remains undetermined. 


\section{About the Authors}

Medical Simulation and Emergency Management Research Group (Drs Rössler Hüpfl, and Schebesta), Department of Anesthesia, General Intensive Care and Pain Management (Drs Rössler, Marhofer, Hüpfl, and Schebesta), Medical University, Vienna, Austria; and Swiss Tropical and Public Health Institute and University of Basel, Basel, Switzerland (Ms Peterhans).

Address correspondence and reprint requests to Bernhard Rössler, $\mathrm{MD}, \mathrm{MIH}$, Department of Anesthesia, Sir Charles Gairdner Hospital, Hospital Avenue, Nedlands 6009,WA, Australia (e-mail: bernhard.roessler@meduniwien.ac.at).

\section{Acknowledgments}

The anesthesiologists who participated in the study have given their time, effort, and dedication to patients worldwide, regardless of nationality, religious belief, or ethnicity. The participating organizations provided their support to this project.

\section{Funding and Support}

This work was supported solely by the Medical University of Vienna, Austria.

\section{Disclaimer}

Bernadette Peterhans has received honoraria from the International Committee of the Red Cross for consultancy work and teaching.

\section{Presentations}

Parts of the findings have been presented as a poster at the Resuscitation Congress in Vienna, Austria, October 18-20, 2012.

Published online: May 10, 2013.

\section{REFERENCES}

1. Kano M, Wood MM, Siegel JM, Bourque LB. Disaster research and epidemiology. In: Koenig KL, Schultz $\mathrm{CH}$, eds. Disaster Medicine: Comprehensive Principles and Practices. New York, NY: Cambridge University Press; 2010:3-20.

2. Salama P, Spiegel P, Talley L, Waldman R. Lessons learned from complex emergencies over past decade. Lancet. 2004;364:1801-1813.

3. McCunn M, Ashburn MA, Floyd TF, et al. An organized, comprehensive, and security-enabled strategic response to the Haiti earthquake: a description of pre-deployment readiness preparation and preliminary experience from an academic anesthesiology department with no preexisting international disaster response program. Anesth Analg. 2010;111:1438-1444

4. Missair A, Gebhard R, Pierre E, et al. Surgery under extreme conditions in the aftermath of the 2010 Haiti earthquake: the importance of regional anesthesia. Prehosp Disaster Med. 2010;25:487-493.

5. Paix BR, Capps R, Neumeister G, Semple T. Anesthesia in a disaster zone: a report on the experience of an Australian medical team in Banda
Aceh following the Boxing Day tsunami. Anaesth Intensive Care. 2005;33:629-634.

6. Rice MJ, Gwertzman A, Finley T, Morey TE. Anesthetic practice in Haiti after the 2010 earthquake. Anesth Analg. 2010;111:1445-1449.

7. Ursprung T. Management of war injuries from the anesthesiologic point of view: a report of experiences from the IKRK hospital in Kabul, September 1990. Z Unfallchir Versicherungsmed. 1991;84:48-55.

8. Baker DJ. The anaesthesiologist and natural disasters. Anesth Int. 2011; 5:10-15.

9. Allcock E, Spencer E, Frazer R, Applegate G, Buckenmaier C III. Continuous transversus abdominis plane (TAP) block catheters in a combat surgical environment. Pain Med. 2010;11:1426-1429.

10. Suzuki T. The situation of anesthesia in Afghanistan. Masui. 2001; 50:805-809.

11. Kaplowitz MD, Hadlock TD, Levine R. A comparison of web and mail survey response rates. Public Opin Q. 2004;68:94-101.

12. Beydon L, Ledenmat PY, Soltner C, et al. Adverse events with medical devices in anesthesia and intensive care unit patients recorded in the French safety database in 2005-2006. Anesthesiology. 2010;112: 364-372.

13. Cooper JB, Newbower RS, Kitz RJ. An analysis of major errors and equipment failures in anesthesia management: considerations for prevention and detection. Anesthesiology. 1984;60:34-42.

14. Orser BA, Chen RJB, Yee DA. Medication errors in anesthetic practice: a survey of 687 practitioners. Can J Anaesth. 2001;48:139-146.

15. Orser BA, Byrick R. Anesthesia-related medication error: time to take action. Can J Anesth. 2004:51:756-760.

16. Gaba DM. Anaesthesiology as a model for patient safety in health care. BMJ. 2000;320:785-788.

17. Mellin-Olsen J, O'Sullivany E, Baloghz D, et al. Guidelines for safety and quality in anesthesia practice in the European Union. Eur J Anaesthesiol. 2007;24:479-482.

18. Merry AF, Cooper JB, Soyannwo O, Wilson IH, Eichhorn JH. International standards for a safe practice of anesthesia 2010. Can J Anaesth. 2010;57:1027-1034.

19. Giannou C, Baldan M. Anesthesia and analgesia in war surgery. In: Giannou C, Baldan M, eds. War Surgery. Geneva, Switzerland: International Committee of the Red Cross Publications; 2009:309-317.

20. Marhofer P, Harrop-Griffiths W, Kettner SC, Kirchmair L. Fifteen years of ultrasound guidance in regional anesthesia: part $1 \mathrm{Br} J$ Anaesth. 2010;104:538-546.

21. du Mortier S, Arpagaus M. Quality improvement programme on the frontline: an International Committee of the Red Cross experience in the Democratic Republic of Congo. Int J Qual Health Care. 2005;17: 293-300.

22. Rahardjo E, Wiroatmodjo K, Koeshartono P. Toward more efficient multinational work on rescue and aid for disasters: lessons learned during the Aceh tsunami and Yogya earthquake. Prehosp Disaster Med. 2008; 23:301-304.

23. Ginosar Y, Shapira SC. The role of an anaesthesiologist in a field hospital during the cholera epidemic among Rwandan refugees in Goma. Br J Anaesth. 1995; 75:810-816. 